\title{
A NEW HIGH-PRECISION CORRECTION METHOD OF TEMPERATURE DISTRIBUTION IN MODEL STELLAR ATMOSPHERES
}

\author{
A. Sapar, R. Poolamäe and L. Sapar \\ Tartu Observatory, 61602 Tõravere, Estonia; sapar@aai.ee
}

Received: 2013 January 9; accepted: 2013 March 27

\begin{abstract}
The main features of the temperature correction methods, suggested and used in modeling of plane-parallel stellar atmospheres, are discussed. The main features of the new method are described. Derivation of the formulae for a version of the Unsöld-Lucy method, used by us in the SMART (Stellar Model Atmospheres and Radiative Transport) software for modeling stellar atmospheres, is presented. The method is based on a correction of the model temperature distribution based on minimizing differences of flux from its accepted constant value and on the requirement of the lack of its gradient, meaning that local source and sink terms of radiation must be equal. The final relative flux constancy obtainable by the method with the SMART code turned out to have the precision of the order of $0.5 \%$. Some of the rapidly converging iteration steps can be useful before starting the high-precision model correction. The corrections of both the flux value and of its gradient, like in Unsöld-Lucy method, are unavoidably needed to obtain high-precision flux constancy. A new temperature correction method to obtain high-precision flux constancy for plane-parallel LTE model stellar atmospheres is proposed and studied. The non-linear optimization is carried out by the least squares, in which the Levenberg-Marquardt correction method and thereafter additional correction by the Broyden iteration loop were applied. Small finite differences of temperature $\left(\delta T / T=10^{-3}\right)$ are used in the computations. A single Jacobian step appears to be mostly sufficient to get flux constancy of the order $10^{-2} \%$. The dual numbers and their generalization - the dual complex numbers (the duplex numbers) - enable automatically to get the derivatives in the nilpotent part of the dual numbers. A version of the SMART software is in the stage of refactorization to dual and duplex numbers, what enables to get rid of the finite differences, as an additional source of lowering precision of the computed results.
\end{abstract}

Key words: stars: atmospheres - stars: early-type - stars: fundamental parameters

\section{A GLANCE AT MILESTONES OF TEMPERATURE CORRECTION METHODS OF MODEL STELLAR ATMOSPHERES}

About six decades ago A. Unsöld in his pioneering paper (Unsöld 1951) and in his excellent monograph (Unsöld 1955) proposed the first version of formula for the temperature correction in the plane-parallel model stellar atmosphere. He 
elaborated a method for the correction of the grey model stellar atmospheres. This method is very simple and it was well solvable without computers, and no integration over the frequencies has been needed. The method improves the temperature distribution using the modeled radiative flux departures from its planned constant value for stationary (time-independent) stellar atmospheres with premeditated effective temperature and pure radiative energy transfer, i.e., without any convective energy transfer in star.

Unsöld introduced three correction terms of different nature. The first of them is the requirement that the total emergent flux must correspond to the assumed effective temperature of the black body. This is a boundary condition for the problem of the radiative energy transfer. It can be ignored, what only somewhat slows down the convergence of iterations.

The second requirement is that in each layer of stellar atmosphere the condition must be hold that the source and the sink terms are equal, i.e., the local flux gradient everywhere in stellar atmosphere equals to zero.

The third requirement is that the model-computed radiative pressure gradient throughout the atmosphere must correspond to the premeditated total flux. This is the integral aspect of the flux constancy.

Thereafter during decades different attempts have been made to modify this iterative model atmosphere correction scheme in order to get higher precision or to diminish the time consumption in essentially more complicated cases of the nongray model atmosphere computations, which demand pan-spectral integrations and more complicated algorithms.

A step forward in the theory was made by taking into account that in the model iteration steps the ratios of the Eddington moments and the ratios of differently averaged opacity coefficients stay almost unchanged (Lucy 1964). Corresponding improved method of the iterative model correction is referred to as the UnsöldLucy method. It has been successfully used in somewhat different forms in widely used stellar model atmosphere codes.

A very important role in the history of stellar atmospheres modeling has played the Kurucz program ATLAS (Kurucz 1970). In the PhD thesis the formulae used and the methods applied in the software have been described in detail and the software has been $a b$ initio publicly available. The most important modernized versions of ATLAS are ATLAS9 (Castelli \& Kurucz 2003), where the opacity distribution function (ODF) has been used, and ATLAS12, where a version of the opacity sampling (OS) has been used for the contribution of spectral lines. A short user manual of ATLAS12 and its comparison with ATLAS9 has been published by Castelli (2005). The more detailed user's guide instructions of ATLAS12 are given at the Kurucz website (http://kurucz.harvard.edu). The method of model temperature correction by Kurucz is an original version related to the Unsöld method. In recent paper by Kirby (2011) a grid of ATLAS9 model stellar atmospheres has been computed for stars having $3500 \leq T_{\text {eff }} \leq 8000 \mathrm{~K}$, i.e. for stars having convective zone in the lower layers of their atmospheres. It has been mentioned that after 30 iterations infrequently differences in flux reached $1 \%$ and in its gradient source and sink terms $10 \%$.

A promising step was the proposal of the method of complete linearization by Mihalas \& Auer (1969). The method can be considered as a generalized NewtonRaphson method. Its drawback is that it demands to find derivatives relative to all model variable values, what results in obtaining a bulky system of linear formulae 
to be solved in order to obtain the corrections. This also makes the computer codes much more complicated. A good review of the formulae, needed in this method, was given by Sakhibullin (1997).

The method of complete linearization has been used for the iterative correction of model stellar atmospheres in the MARCS code, planned primarily for computing model atmospheres for relatively cool stars. A detailed summary of the methods used in the MARCS code and the milestones of its development was described by Gustafsson et al. (2008). In this code the process of iteration is terminated, if the corrections of flux and its gradient for all model layers are less than $1.5 \%$.

The Tübingen version of computing model stellar atmospheres, TMAP, has been composed by Werner and Rauch primarily for NLTE modeling but in it a version of the Unsöld-Lucy method for iterative correction of the plane-parallel atmospheres is also enabled. The process of iteration is terminated, if the relative temperature corrections for all model layers are less than $10^{-6}$, or 100 iterations have been made. The result seems promising for high-precision modeling, but the small temperature corrections can correspond to essentially lower precision of the flux and its gradient. The final version of the TMAP package is accessible ${ }^{1}$. The classical stellar atmosphere problem of computing precise model stellar atmospheres and their numerical implementations, based on the PRO2 software, have been formulated and analyzed in detail by Werner \& Dreizler (1999).

A software TLUSTY for computation of model stellar atmospheres was elaborated by Hubeny and thereafter updated and essentially improved in collaboration with colleagues, especially with Lanz. In this package the hybrid method of the complete linearization and accelerated lambda iteration has been applied to solve all basic equations. The process of iteration is conventionally terminated, if the relative corrections for all model layers are less than $0.1 \%$. The user's guide composed by Hubeny and Lanz for the last version of TLUSTY package is accessible ${ }^{2}$.

A very sophisticated computer code for modeling of stellar atmospheres is PHOENIX (Hauschildt et al. 2003), which enables both the spherical and planeparallel modeling. In it a version of the Unsöld-Lucy temperature correction method was used and tested. It was concluded that in addition to the low memory requirements and low time consumption the Unsöld-Lucy method is more stable than the method of complete linearization. This computer code with large and detailed input physics and wide field of application has been oriented to parallel computations on supercomputers. In it mostly the scheme of nested iterations is used, which reduces the high-dimensional linearization problem to sets of smaller dimensional iterations. A special item is the method of the temperature correction.

All the codes described above are written in different revisions of Fortran and most of the programs are only partially accessible to astrophysical community.

\section{STRATEGY OF HIGH-PRECISION MODELING OF STELLAR ATMOSPHERES BY SMART}

Presently the time consumption even on usual personal computers is not a crucial problem, but simplicity of the formulae used and of the software will always remain welcomed in order to get a better understanding of physical processes and laws governing the model atmospheres.

\footnotetext{
1 http://astro.uni-tuebingen.de/ rauch/

2 http://tlusty.gsfc.nasa.gov
} 
By our SMART (Stellar Model Atmospheres and Radiative Transport) software computations we established, that the Unsöld-Lucy method does not give highprecision constancy of the flux, what is needed to be sure that the method is selfconsistent. Further we describe a version of the Unsöld-Lucy method which has been currently used by us to compute a grid of hot model stellar atmospheres. We concluded that the method gives a good accuracy (about $0.5 \%$ in the radiative flux constancy), but it does not converge to high-precision results. In addition, as usual, a damping factor about $0.5-0.7$ must be used in order to avoid overcorrection, giving slowly damping oscillatory iterative steps.

Our high-precision correction method is a version of the non-linear optimization procedure, based on the least-squares cost function relative to both the radiative flux and its gradient. In this aspect there is an analogy with the Unsöld-Lucy method. The Jacobian is obtained by the Levenberg-Marquardt method (also known as the damped Newton's method), where in addition to the cost function a quadratic penalty term has been added. The Levenberg-Marquardt method belongs to the class of nonlinear optimization methods. Using the $0.1 \%$ variations for the temperature values subsequently in each atmospheric layer, we have used the method of finite differences for computing the derivatives. As a rule, single Jacobian cycle gives the high flux constancy to about $10^{-2} \%$.

Using the finite difference method does not sophisticate the computer code as in the case of complete linearization method. Happily we realized that the finite difference method can be replaced by a tricky refactorization of the software. Such perspective is opened when we take into use the dual numbers which similarly to complex numbers consist of two components, the real component and the so-called infinitesimal component. Differently from the complex numbers, the real and the infinitesimal parts in computations avoid mixing. Nilpotency of infinitesimal parts generates important property of these numbers that they enable to obtain automatically exact values of the derivatives, corresponding to the used algorithms, in the infinitesimal parts of dual numbers.

If the used algorithms contain the complex numbers, as in the case of the absorption coefficient expressed via the complex number special functions (Sapar \& Poolamäe 2012), there appears inevitable necessity to generalize the dual numbers also for the case of complex number algorithms. A Fortran module with corresponding data types, overloaded operators and functions has been composed. The dual complex numbers we call the duplex numbers.

At present we are preparing to apply for computations the Levenberg-Marquardt method by the use of the duplex numbers, refactorizing a version of the SMART software.

\section{A VERSION OF THE TRADITIONAL TEMPERATURE CORRECTION}

In order to obtain a vision about the temperature correction method used in our Fortran90/95 code SMART, we present here the last version of the Unsöld-Lucy method used.

The equation of the radiative transfer for plane-parallel stellar atmospheres has the form

$$
\mu \frac{d I_{\nu}}{d r}=\kappa_{\nu} S_{\nu}-\kappa_{\nu} I_{\nu}
$$

In this equation the opacity coefficient $\kappa_{\nu}$ incorporates the first order radiative processes - absorption and emission, which are monochromatic in radiative trans- 
fer, but also the scattering as the second order radiative process, according to the classification of Feynman diagrams. The absorption and emission processes are easily thermalized (the LTE approximation), and this is taken into account in the Einstein spontaneous and induced transition coefficients. The scattering takes into account the non-thermalized processes. Thus, we can write

$$
\kappa_{\nu}=\alpha_{\nu}+\sigma_{\nu \nu^{\prime}}
$$

The source function $S_{\nu}$ is given by

$$
\kappa_{\nu} S_{\nu}=\alpha_{\nu} B_{\nu}+\sigma_{\nu \nu^{\prime}} J_{\nu^{\prime}}
$$

In this equation and further, integration is carried out over the primed indices and corresponding polar angles. From Equation (1) in this way for the first Eddington moment, $H$, being the Eddington radiation flux, we obtain

$$
\frac{d H}{d r}=\alpha_{B} B-\alpha_{J} J
$$

Here the terms with the isotropic scattering coefficient have cancelled.

For the second Eddington moment $K$ we obtain

$$
\frac{d K}{d r}=-\kappa_{H} H
$$

Physical meaning of the Eddington moments and the corresponding absorption and opacity coefficients should be familiar without any additional explication.

The equation of hydrostatic equilibrium for the stellar matter in the simplest way can be written in the form

$$
\frac{d P}{d m}=g
$$

where $m$ is the mass column density. Further, the total pressure is given by $P=P_{g}+\frac{4 \pi}{c}\left(K-K^{0}\right)+P_{\text {turb }}$, where $P_{\text {turb }}$ corresponds to microturbulence.

The strategy of further study starts from the due choice of the optical depth. Usually the Rosseland mean absorption coefficient has been used for the definition of the mean optical depth. It has the advantage that it can be computed also without modeling of stellar atmosphere. In this aspect it can be treated as a local characteristic. The same advantage is in computation of the Planck mean absorption coefficient $\kappa_{B}$, which has been used by some investigators for the definition of optical depth. However, both of them have the drawback that they are not connected directly and simply to the radiation field in stellar atmosphere. The best method for model atmosphere correction is to specify the optical depth $\tau$ as the flux opacity, namely by $\kappa_{H} d r=-d \tau$, giving simply

$$
\frac{d K}{d \tau}=H \text {. }
$$

This equation for the radiation field by its meaning and form is very similar to the Equation (6) of hydrostatic equilibrium for stellar matter.

Equation (4) can now be written in the form

$$
\frac{d H}{d \tau}=\frac{\alpha_{J}}{\kappa_{H}} J-\frac{\alpha_{B}}{\kappa_{H}} B .
$$


Introducing the notations

$$
k_{j}=\frac{\alpha_{J}}{\kappa_{H}} \quad \text { and } \quad k_{b}=\frac{\alpha_{B}}{\kappa_{H}},
$$

we obtain

$$
\frac{d H}{d \tau}=k_{j} J-k_{b} B
$$

Similarly to the ratios of the Eddington moments, the ratios $k_{j}$ and $k_{b}$ can be considered as constants in the temperature correction steps of flux iteration loop. Thus, we discard the corresponding relatively small corrections compared to the corrections of the radiation field. This decelerates somewhat the process of convergence but simplifies the software.

If the microturbulence is ignored, then we can carry out integration of Equation (6), obtaining

$$
P_{g}+\frac{4 \pi}{c}\left(K-K^{0}\right)=g m .
$$

This equation specifies the connection between the gas pressure and the radiation field in stellar atmospheres. In this formula $K^{0}$ is the boundary value of $K$ corresponding to the emergent flux. Further, from (10) it follows that for correct value of $H$ holds

$$
K=K^{0}+H \tau \text {. }
$$

Now we obtain

$$
P_{g}+\frac{4 \pi}{c} H \tau=g m
$$

The computed deviations $\Delta$, defined for the Eddington moments $J, H$ and $K$ by stereotype $\Delta Y=Y-Y_{*}$, where $Y_{*}$ corresponds to the needed value of the quantity, we must compensate by $\Delta B$. The values of arguments $m$ and $\tau$ we treat as the correct ones. Thus, from Equation (7) it follows that

$$
\frac{d \Delta K}{d \tau}=\Delta H
$$

and from (10) we obtain

$$
\frac{d \Delta H}{d \tau}=\frac{d\left(H-H_{*}\right)}{d \tau}=\frac{d H}{d \tau}=k_{j} \Delta J-k_{b} \Delta B .
$$

Further, from (11) it follows that

$$
P_{g}+\Delta P_{g}+\frac{4 \pi}{c}\left(K+\Delta K-K^{0}-\Delta K^{0}\right)=g m .
$$

For the reduction of all corrections to $\Delta H$, we introduce a given function $\gamma$ for the next iteration step and $\chi$ as a known number by

$$
J=3 \gamma K \quad \text { and } \quad J^{0}=2 \chi H^{0} .
$$

This assumption is due to the circumstance that the ratios of Eddington moments are almost constant for each single step of the model correction loop. The quantity 
$\gamma$ has been chosen so that in deep layers of a model atmosphere it tends to unity. The quantity $\chi$ has been chosen so that it is unity for black body emergent flux.

Thus, we obtain

$$
\Delta J=3 \gamma \Delta K \quad \text { and } \quad \Delta J^{0}=2 \chi \Delta H^{0} .
$$

Now we can write

$$
3 \gamma_{0} \Delta K^{0}=2 \chi \Delta H^{0}
$$

and from (12) it follows that

$$
\Delta K=\Delta K^{0}+\int_{0}^{\tau} \Delta H d \tau
$$

To obtain $d H / d \tau=0$, we make in Equation (8) corrections to $J$ and $B$, obtaining

$$
k_{j} J-k_{b} B=-3 \gamma k_{j} \Delta K+k_{b} \Delta B .
$$

Thus, replacing $\Delta K$ into this expression, we get finally for the temperature correction equation

$$
k_{b} \Delta B=2 f k_{j} \Delta H^{0}+k_{j} J-k_{b} B+3 \gamma k_{j} \int_{0}^{\tau} \Delta H d \tau
$$

where $f=\chi \gamma / \gamma_{0}$. Using the notation $R_{b}^{j}=k_{j} / k_{b}$, we obtain

$$
B+\Delta B=R_{b}^{j}\left(2 f \Delta H^{0}+J+3 \gamma \int_{0}^{\tau} \Delta H d \tau\right) .
$$

Taking the column density $m$ as argument, the integral expression can be written in the form

$$
\int_{0}^{\tau} \Delta H d \tau=\int_{0}^{m} \frac{\kappa_{H} \Delta H}{\rho} d m
$$

The simplest way to find the integral is to use instead of the argument $\tau$ the atmospheric layer number $n$. Thus, using (12) we obtain

$$
\int_{0}^{\tau} \Delta H d \tau=\int_{1}^{n} \frac{d K}{d n} \frac{\Delta H}{H} d n
$$

This expression is preferred because its computation is simpler, it does not contain explicitly any absorption coefficient and depends only on the radiation field. In the given formulae all corrections were made, tacitly assuming validity of the condition of local thermodynamical equilibrium (LTE).

After each iteration step, the electron density has been also corrected using $\Delta P_{g}$ from (16) and thereafter the corrected electron pressure from Saha equations. 
As it is evident both from general ideas and model computations, the correction due to the emergent flux $\Delta H^{0}$ and due to the presence of flux gradient diminish quite rapidly inside the model atmosphere, but the integral flux contribution gives essential correction.

The given approach to the iterative correction loop of the model stellar atmosphere was used in our computations by Fortran 90/95 software SMART, using a parallel-processing controlled by the python script, on a small local PC network. Initial rapid convergence of crude initial model, giving a relative error of the order $10 \%$, has slowed down to the final plateau with a relative precision typically of the order of $0.1 \%$ at about 100 iteration loop steps and giving unremovable zigzags at large optical depths (see Figure 1). This demonstrates that there are only small deviations from the self-consistency.

Different possibilities and some results obtained by our SMART software have been described in our former papers (Sapar \& Poolamäe 2003; Aret \& Sapar 2002). General characteristics of the software and different results obtained with it have been reported in papers by Sapar et al. (2007a,b, 2008, 2009) and Aret et al. (2008).

Some of the rapidly converging iteration steps with the Unsöld-Lucy method can be useful to apply before starting with the high-precision model correction method which we propose.

\section{APPLICATION OF THE LEAST SQUARES METHOD FOR HIGH-PRECISION MODELING OF STELLAR ATMOSPHERES}

From numerous model atmoshere computations we concluded that the traditional iteration loop for finding the corrected temperature distribution values of a model stellar atmosphere does not give the desired high-precision final constancy of the flux. Thus, it is important to elaborate a method which ensures a highprecision flux constancy in the best fit self-consistent manner.

Similarly to the Unsöld-Lucy method, by experimentation we have concluded that the method must take into account not only the flux constancy, but also the requirement of local equality of the absorbed and emitted radiation. This means that the method must explicitly incorporate the requirement that the derivative of the flux (10) must be zero everywhere. Studying the Unsöld-Lucy method it has been found that this is essential in order to get adequate centers of strong spectral lines and to get adequate profiles for spectral lines, formed in the uppermost layers of stellar atmospheres. This is an evidence of the requirement that the gradient of the flux must be zero everywhere.

A promising method to get a high-precision flux constancy of model stellar atmospheres is to apply a method of non-linear optimization, namely the method of least squares. To our knowledge, this method has hitherto not been applied to the problem. For compactness of equations we further denote the atmospheric quasi-vectorial (single index) variables by boldface letters and similar quasi-tensors by additional cap above them.

For the present problem we specify the reduced residual $\mathbf{r}$ by

$$
\mathbf{r}=\mathbf{f}-1, \quad \mathbf{f}=\left(\mathbf{H} / H_{\mathrm{eff}}, \mathbf{D}\right), \quad D_{i}=\left.\frac{k_{j} J}{k_{b} B}\right|_{i}
$$

Here $H_{\text {eff }}$ is the value of the Eddington flux (black-body radiative flux per stera- 
dian), corresponding to the effective temperature of the model stellar atmosphere, $\mathbf{H}=\mathbf{H}(\mathbf{T})$ is the computed quasi-vector of the Eddington flux of the model stellar atmosphere, corresponding to the layers $i$ in the atmosphere and $\mathbf{D}$ shows the deviation of the sink/source ratio of the local radiation field in the stellar atmosphere. The model correction quasi-vector $\Delta \mathbf{T}$ (the thermal shift vector) is to be found relative to the previous step temperature distribution by

$$
\mathbf{T}=\mathbf{T}+\Delta \mathbf{T}
$$

The initial cost function $S$ in the local minimum we define in the usual form:

$$
S=\frac{1}{2} \mathbf{r}^{2}
$$

As is seen, $S$ is in fact a $\chi^{2}$-expression for our problem. The presence of the factor $1 / 2$ simplifies somewhat the formulae.

In search of the local minimum point, as the first step we find the gradient of $S$, which can be written in the form

$$
\nabla S=\frac{\partial S}{\partial \mathbf{T}}=\mathbf{r} \nabla \mathbf{f}=\mathbf{r} \hat{\mathbf{J}}
$$

where the matrix components of the $2 N \times N$ dimensional Jacobian, $\hat{\mathbf{J}}$, are defined by

$$
\nabla_{j} f_{i}=\frac{\partial f_{i}}{\partial T_{j}}=\hat{\mathbf{J}}_{i j}
$$

The Jacobian $\hat{\mathbf{J}}$ is needed for finding the least local value of the cost function $S$.

The component values of the Jacobian are to be numerically computed by the SMART software in the loop steps over all $N$ atmospheric layers $j$, successively changing in each model step (by a small rate $\varepsilon$ ) only corresponding single temperature value by $T_{j}:=T_{j}(1+\varepsilon)$. We have accepted $\varepsilon=0.001$.

The next task is to find in an optimum way the thermal shift vector $\Delta \mathbf{T}$. In order to obtain from $S$ the next approximation $S^{+}$, the corrected residual is obtained in the form

$$
\mathbf{r}^{+}=\mathbf{r}(\mathbf{T}+\Delta \mathbf{T})=\mathbf{r}(\mathbf{T})+\hat{\mathbf{J}} \Delta \mathbf{T}
$$

where $\Delta \mathbf{T}$ is the thermal shift vector. The corresponding $S^{+}$takes the form

$$
S^{+}=\frac{1}{2}\left(\mathbf{r}^{+}\right)^{2} .
$$

The gradient of $S^{+}$is

$$
\nabla S^{+}=\mathbf{r}^{+} \hat{\mathbf{J}}
$$

To minimize $S^{+}$, its gradient expression is set to zero, obtaining the GaussNewton minimizer of shift vector $\Delta \mathbf{T}$ as the solution of the obtained linear system of equations

$$
\mathbf{r}^{+} \hat{\mathbf{J}} \equiv \mathbf{r} \hat{\mathbf{J}}+\hat{\mathbf{H}} \Delta \mathbf{T}=0 .
$$

Here the quadratic $N \times N$ Hessian matrix has the form

$$
\hat{\mathbf{H}}=\hat{\mathbf{J}}^{T} \hat{\mathbf{J}} \text {. }
$$


The matrices with superscript $T$, say as $\hat{\mathbf{J}}^{T}$ here, means the transposition of its columns and lines.

The Levenberg-Marquardt method (Levenberg 1944; Marquardt 1963), which we use, starts by adding to the cost function a Lagrangian penalty term in the form

$$
S=\frac{1}{2} \mathbf{r}^{2}+\frac{1}{2} \gamma(\Delta \mathbf{T})^{2}
$$

where $\gamma$ is a small positive number (the penalty rate or the damping term), the numerical value of which is usually accommodated by a corresponding software procedure. From this cost function one obtains the equation for finding the shift vector in the form

$$
\hat{\mathbf{H}} \Delta \mathbf{T}+\mathbf{r} \hat{\mathbf{J}}+\gamma \Delta \mathbf{T}=0 .
$$

This system of equations with additional damping term enables in different ways to find the next recurrent steps to minimize the cost function. An efficient method to minimize $S$ is the Broyden's method, which can be treated as a generalized Newton secant method for finding recurrently the corrections to the solution of the Levenberg-Marquardt system of equations.

The Broyden method starts from solving the Levenberg-Marquardt equation with the computed initial temperatures $\mathbf{T}_{0}=\mathbf{T}$, the initial residual $\mathbf{r}_{0}=\mathbf{r}$ values and the corresponding computed matrices $\hat{\mathbf{H}}_{\mathbf{0}}=\hat{\mathbf{H}}, \hat{\mathbf{J}}_{\mathbf{0}}=\hat{\mathbf{J}}$.

Instead of starting a new Levenberg-Marquardt iteration loop for computing the new values of Jacobian and Hessian, the Broyden method starts from correcting the value of Jacobian in each single iteration step, finding also all the quantities, which are needed for the next iteration step. The Broyden loop starts at $k=0$.

In each step of the Broyden loop first the temperature shift for the step, $\mathbf{t}=$ $\Delta \mathbf{T}_{k+1}$, is found from the system of linear equations

$$
\hat{\mathbf{H}}_{k} \mathbf{t}+\mathbf{r}_{k} \hat{\mathbf{J}}_{k}+\gamma \mathbf{t}=0, \quad \hat{\mathbf{H}}_{k}=\hat{\mathbf{J}}_{k}^{T} \hat{\mathbf{J}}_{k} .
$$

Thereafter the corrected temperature $\mathbf{T}_{k+1}=\mathbf{T}_{k}+\mathbf{t}$ is found and used to compute by SMART the corresponding residual $\mathbf{r}_{k+1}$.

Broyden's rank one recurrent updating (Broyden 1965) is given by a simple formula

$$
\hat{\mathbf{J}}_{k+1}=\hat{\mathbf{J}}_{k}+\mathbf{U}_{k} \times \mathbf{t}^{T},
$$

where $\times$ means direct product of vectors and

$$
\mathbf{U}_{k}=\frac{1}{\mathbf{t}^{T} \mathbf{t}}\left(\mathbf{r}_{k+1}-\mathbf{r}_{k}-\hat{\mathbf{J}}_{k} \mathbf{t}\right) .
$$

At last we find also the new Hessian by $\hat{\mathbf{H}}_{k+1}=\hat{\mathbf{J}}_{k+1}^{T} \hat{\mathbf{J}}_{k+1}$. Thereafter the following Broyden iteration step is to be started by substitution $k:=k+1$.

An important feature of the Broyden loop is that for $N$ arguments (in the present case the number of atmospheric layer points) it gives excellent fit in $N$ iteration steps. For the present least squares expression this means that the Broyden method gives in $N$ iteration steps very high flux constancy in the model stellar atmospheres. The model computations carried out showed that the flux constancy obtained is of the order of $10^{-2} \%$. The precision of the local ratio of emitted and absorbed radiation energy is of the same order. Application of the method has 


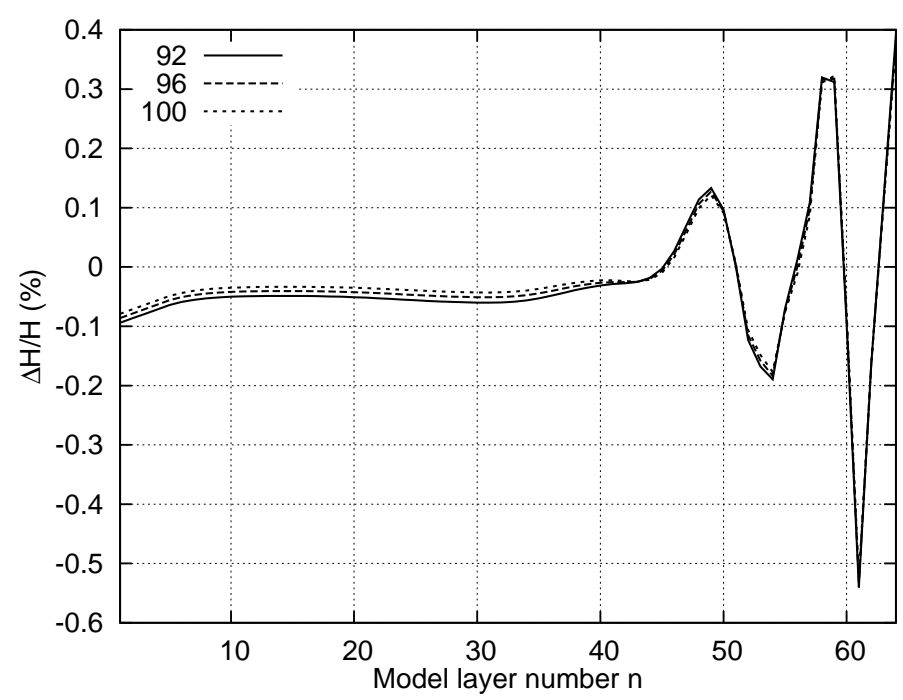

Fig. 1. A typical picture of the final relative precision of the radiative flux obtained using the Unsöld-Lucy correction method (in \%). As argument values in all Figures the model layers, numerated starting from outside, are given. Model parameters: $T_{\text {eff }}=$ $10750 \mathrm{~K}, \log g=4$. The legend inside the frame gives the iteration numbers.

shown that if we start from the former Kurucz model atmospheres, which give low flux constancy for our model atmospheres, then high precision has been obtained by the initial Jacobian with following no more than $N$ steps of Broyden rank one updating, each giving a new Jacobian and corresponding Hessian for the next iterative step of the temperature corrections by the Levenberg-Marquardt method. The Fortran90/95 software SMART has been controlled by Python script organizing the parallel-processing tasks to slaves and master using the local PC network. The integrated Levenberg-Marquardt part is implemented in $\mathrm{C}++$ using in it the code from Lourakis (2004), which has been slightly modified by us.

In Figure 1 we present a typical picture of the final precision of the radiative flux, obtained by us using the Unsöld-Lucy method of its iterative correction. In Figure 2 we demonstrate how effectively the first Levenberg-Marquardt least squares step improves the flux constancy of a model atmosphere. In Figure 3 we show its improvement in the consequent Broyden loop steps. Figure 4 shows typical final curves of the flux constancy when the Levenberg-Marquardt method + Broyden loop has been applied. Notice that in the deeper atmospheric layers at all model effective temperatures the flux has very high precision. However, the ratio of the absorbed and emitted radiation (Figure 5) shows a small excess in deep layers and a small deficiency in outer layers from which the emergent flux escapes. 


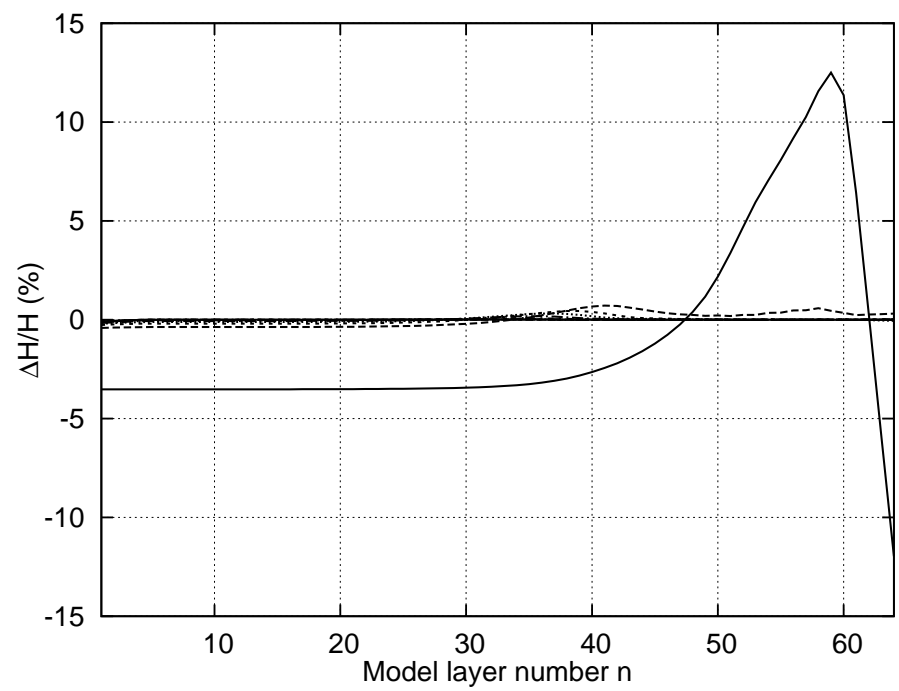

Fig. 2. The relative initial stellar model atmosphere flux (solid line) and its consequent iterative improvement by the Broyden loop steps (in \%). The first correction is obtained using the Jacobian, computed by the Levenberg-Marquardt method and improving it by iterations of the Broyden loop. The model parameters: $T_{\text {eff }}=16000 \mathrm{~K}, \log g=4$.

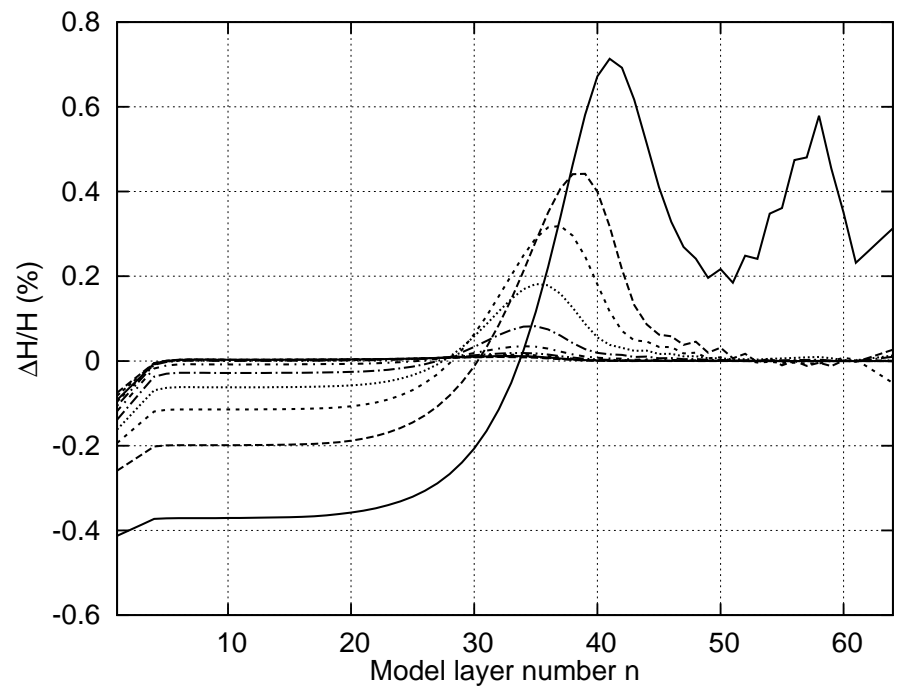

Fig. 3. Zoomed Figure 2, from where the initial model atmosphere flux has been removed. The deviations (in \%) of the stellar model atmosphere flux, obtained using the Jacobian computed by the first cycle of the Levenberg-Marquardt method (solid line) and in its consequent iterative improvement by the Broyden loop steps. The model parameters: $T_{\text {eff }}=16000 \mathrm{~K}, \log g=4$. 


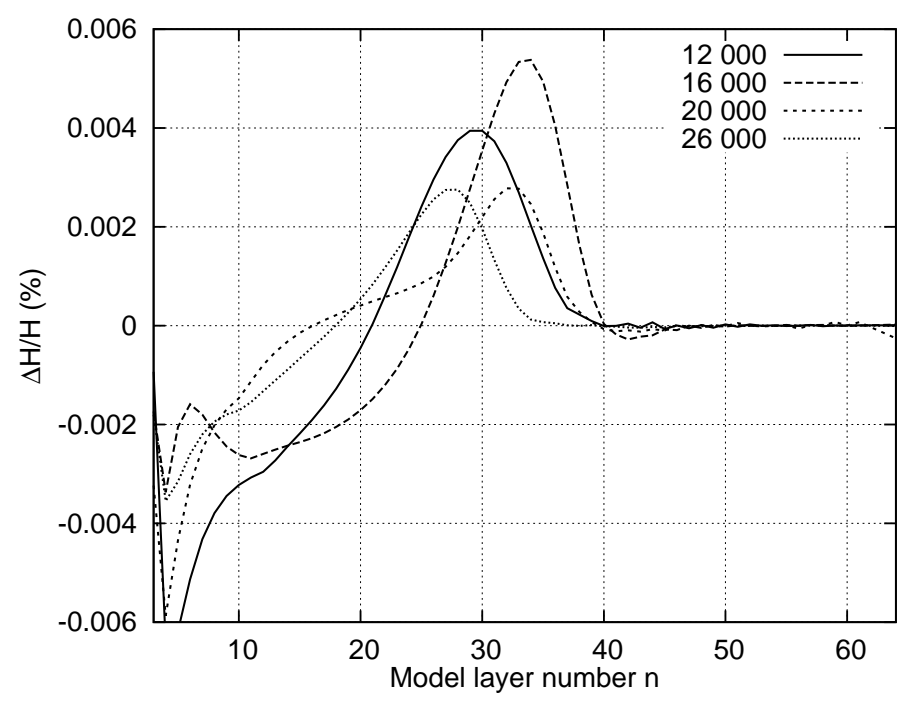

Fig. 4. The final high-precision constancy of the model stellar atmosphere flux (in $\%$ ), obtained computing the Jacobian by Levenberg-Marquardt method and thereafter improving it by iterations of the Broyden loop. In the models $\log g=4$ and in the legend - the effective temperatures.

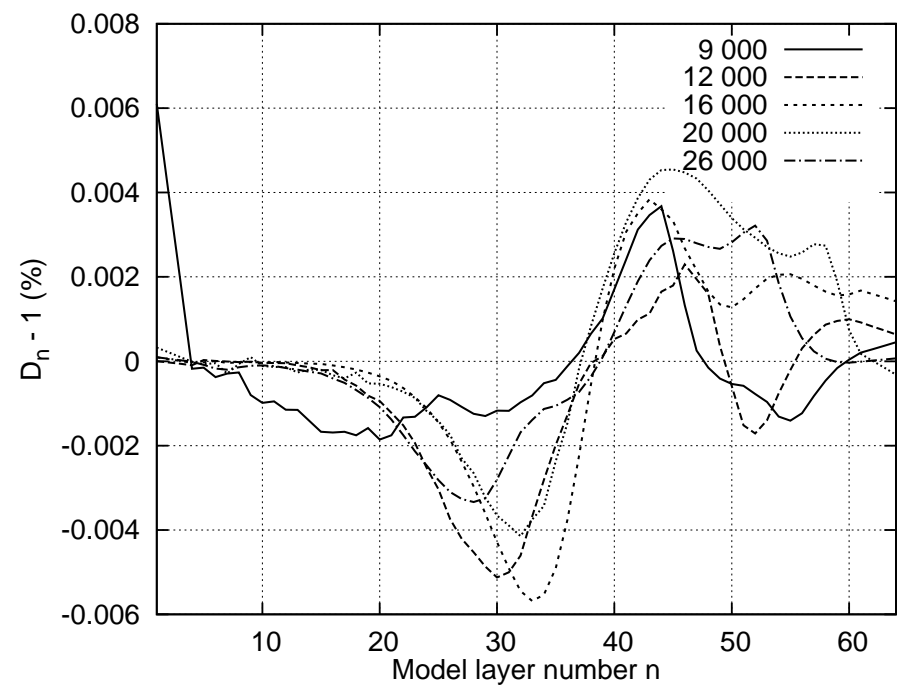

Fig. 5. The final high-precision local ratio of the absorbed and radiated radiative flux (in \%). The result is obtained computing the Jacobian by the Levenberg-Marquardt method and thereafter improving it by iterations of the Broyden loop. In the models $\log g=4$ and in the legend - the effective temperatures. 


\section{CONCLUSIONS}

The iterative correction of the temperature distribution in stellar atmospheres by different versions of the Unsöld-Lucy method has very low memory requirement, but the iterative procedure of its application converges to flux constancy with insufficient precision. This method has been applied hitherto in several software packages for stellar model corrections.

The other method is a complete linearization which, when analytically applied, demands essential additional subroutines, makes the software much more complicated and generates bulky systems of linear equations for the determination of the corrections. This method can be treated as a version of the generalized Newton-Raphson method of iterative improvement of the temperature and pressure distribution in stellar atmospheres.

We conclude that for correcting of the plane-parallel model stellar atmospheres the Levenberg-Marquardt method, belonging to the domain of the nonlinear optimization, applied together with the Broyden iteration loop, is an effective method to obtain the high-precision flux constancy, which is about two orders of magnitude superior relative to the traditional Unsöld-Lucy method, and the time consumption for both methods is comparable. Usually one Jacobian step is sufficient to get the relative flux constancy of the order $10^{-2} \%$. This means that the method is highly self-consistent. The results are visualized in Figures 1-5. A parallel-processing by the Python script has been used in the SMART software by suitably dividing the total spectral interval used in the modeling. We are busy to refactorize the SMART software for applying the dual numbers in the Levenberg-Marquardt method thus avoiding the possible loss of precision due to finite differences.

As a concluding remark it deserves to mention that similar methods have been used by other authors for study of somewhat different problems. For instance, the Jacobian and Broyden algorithm as additional accelerating method in the non-LTE radiation transfer computations by ALI (Approximate Lambda Iteration) have been used by Koesterke et al. (1992). Similarly, using the linearized perturbation method for the temperature-correction of non-LTE modeling of stellar atmospheres and applying the ALI method for radiation transfer, Werner et al. (2003) have studied the iterative correction of model stellar atmospheres, describing in detail the derivation of the sophisticated formulae used.

ACKNOWLEDGMENTS. This work was supported by the research project SF0060030s08 of the Estonian Ministry of Education and Research.

\section{REFERENCES}

Aret A., Sapar A. 2002, AN, 323, 21

Aret A., Sapar A., Poolamäe R., Sapar L. 2008, in The Art of Modelling Stars in the 21st Century, IAU Symp. 252, 41

Broyden C. G. 1965, Math. Comp., 19, 577

Castelli F., Kurucz R. L. 2003, in Modelling of Stellar Atmospheres, IAU Symp. 210, Poster A20; arXiv:astro-ph/0405087v1, 2004

Castelli F. 2005, MmSAI, 8, 25

Gustafsson B., Edvardsson B., Eriksson K. et al. 2008, A\&A, 486, 951

Hauschildt P. H., Barman T. S., Baron E., Allard F. 2003, in Stellar Atmosphere Modeling, ASPC, 228, 227 
Kirby E. N. 2011, PASP, 123, 531

Koesterke L., Hamann W.-R., Kosmol P. 1992, A\&A, 255, 490

Kurucz R. L. 1970, ATLAS: A Computer Program for Calculating Model Stellar Atmospheres, SAO Special Report, No. 309

Levenberg K. 1944, Quart. Appl. Math., 2, 164

Lourakis M.I.A. 2004, http://www.ics.forth.gr/ lourakis/levmar/

Lucy L. B. 1964, SAO Special Report, No. 167, 93

Marquardt D. 1963, J. Soc. Indust. Appl. Math., 11 (2), 431

Mihalas D., Auer L. H. 1969, ApJ, 158, 641

Sakhibullin N. 1997, Methods of Modelling in Astrophysics, Fen Press, Kazan (in Russian)

Sapar A., Poolamäe R. 2003, in Stellar Atmosphere Modeling, ASPC, 288, 95

Sapar A., Aret A., Sapar L., Poolamäe R. 2007a, in Spectroscopic Methods in Modern Astrophysics, INASAN, p. 220 (in Russian)

Sapar A., Poolamäe R., Sapar L., Aret A. 2007b, in Spectroscopic Methods in Modern Astrophysics, INASAN, p. 236 (in Russian)

Sapar A., Aret A., Sapar L., Poolamäe R. 2008, in Precision Spectroscopy in Astrophysics: ESO Astrophysics Symposia, Lisbon Aveiro, p. 145

Sapar A., Aret A., Sapar L., Poolamäe R. 2009, New Astronomy Reviews, 53, 240

Sapar A., Poolamäe R. 2012, Baltic Astronomy, 21, 243

Unsöld A. 1951, Naturwiss., 38, 525

Unsöld A. 1955, Physik der Sternatmosphären, Springer-Verlag

Werner K., Dreizler S. J. 1999, Comput. Appl. Math., 109, 65

Werner K., Deetjen J. L., Dreizler S. et al. 2003, ASPC, 288, 31 